$\underline{\mathbf{P}-196}$

\title{
Alkaloids from the Stems of Enicosanthellum Pulchrum (King) Heusden
}

\author{
Juriyati Jali1 ${ }^{1, *}$, Ooi Ying Xiang ${ }^{2}$, Khairana Husain ${ }^{1}$, Jamia Azdina Jamal ${ }^{1}$, Hazni Falina Mohamad ${ }^{2}$
} and Kartiniwati Muhammad ${ }^{2}$

\footnotetext{
${ }^{I}$ Drug and Herbal Research Centre, Faculty of Pharmacy, Universiti Kebangsaan Malaysia,Jalan Raja Muda Abdul Aziz, 50300 Kuala Lumpur; ${ }^{2}$ Faculty of Pharmacy, Universiti Kebangsaan Malaysia, Jalan Raja Muda Abdul Aziz,50300 Kuala Lumpur; E-mail: juriyati@pharmacy.ukm.my
}

Enicosanthellum pulchrum (King) Heusden belongs to the plant family Annonaceae, is a coniferous tree with green flowers. This study was carried out to isolate and identify the chemical constituents present in the methanol extract of E. pulchrum stems. The plant sample was extracted with hexane, ethyl acetate and methanol successively using Soxhlet apparatus. The methanol extract was then subjected to vacuum liquid chromatography eluted with gradient solvent systems of hexane-ethyl acetate (100:0-0:100) and ethyl acetate-methanol (100:0-0:100) to give eight fractions (I-VIII). Repeated column chromatography of fraction II has led to the isolation of three alkaloids, namely, liriodenine, lysicamine and liridine. Structure elucidation of the isolated compounds was accomplished by means of spectroscopic techniques, as well as by comparison of their spectral data with those reported in the literature.

Keywords: Enicosanthellum pulchrum, Annonaceae, Alkaloids, Liriodenine, Lysicamine, Liridine. 